\title{
Intervención psicológica de un Trastornos de la Conducta Alimentaria abordado desde la Terapia Cognitivo Conductual: Presentación de un caso de Anorexia Nerviosa
}

\section{Psychological intervention of Eating Disorders approached from Behavioral Cognitive Therapy: Presentation of a case of Anorexia Nervosa}

\author{
Fecha de recepción: 17-07-2018
}

Fecha de aceptación: 18-07-2018
Begoña Martínez Amores

Centro Crea

\section{resumen/ahstract:}

En el presente artículo se expone un procedimiento de intervención psicológica cognitivo-conductual aplicado a una adolescente de 13 años con un trastorno del comportamiento alimentario, diagnosticada con Anorexia Nerviosa. Se utilizó un diseño de caso único y medidas pre-post, con cuestionarios de patología alimentaria como el EAT40, BULIT-R, la escala YBC-EDS y el BSQ, y de bienestar emocional como el STAI y el BDI-II. La intervención fue diseñada a partir del análisis funcional del comportamiento con el fin de: aumentar la motivación y adherencia al tratamiento, establecer un patrón de conducta alimentario saludable, estabilizar el estado de ánimo y mejorar el autoconcepto. Las técnicas empleadas fueron: psicoeducación, técnicas motivacionales, técnicas conductuales, técnicas de exposición y reestructuración cognitiva. La intervención se llevó a cabo en 18 meses, en los que las sesiones eran dobles semanalmente durante los primeros 8 meses y posteriormente las sesiones fueron semanales. A partir de un análisis cualitativo y cuantitativo de la evolución de la paciente, se puede concluir que el tratamiento fue eficaz.

In the present article is presented a cognitive-behavioral psychological intervention procedure applied to a 13-year-old adolescent with an eating behavior disorder, diagnosed with Anorexia Nervosa. We used a unique case design and pre-post assessments, with eating pathology questionnaires such as the EAT4O, BULIT-R, the YBC-EDS scale and the $B S O$, and emotional well-being such as STAI and BDI-II. The intervention was designed based on the functional analysis of behavior in order to: increase motivation and adherence to treatment, establish a healthy eating behavior pattern, stabilize mood and improve self-concept. The techniques used were: psychoeducation, motivational techniques, behavioral techniques, exposure techniques and cognitive restructuring. The intervention was carried out in 18 months, in which the sessions were double twice a week during the first 8 months and then the sessions were weekly. From a qualitative and quantitative analysis of the evolution of the patient, it could be concluded that the treatment was effective.

\section{palabras clave/keywords:}

Estudio de caso único, trastorno del comportamiento alimentario, intervención cognitivo-conductual, anorexia nerviosa. Single case study, eating disorder, cognitive-behavioral intervention, anorexia nervosa. 


\section{Introducción}

Los trastornos de la conducta alimentaria (TCA) son enfermedades psiquiátricas complejas, con etiología multicausal, que afectan principalmente a adolescentes y mujeres jóvenes. El diagnóstico más frecuente durante la adolescencia es el de TCA no especificado, seguido por el de anorexia nerviosa y bulimia nerviosa (López y Treasure, 2011). Todos estos trastornos comparten síntomas cardinales tales como la preocupación excesiva por la comida, peso y/o figura corporal y el uso de medidas no saludables para controlar o reducir el peso corporal (APA, 2013).

El Manual Diagnóstico y Estadístico de los Trastornos Mentales (DSM-5), (APA, 2013) ha establecido en su última edición tres trastornos principales dentro de la categoría TCA: anorexia nerviosa (AN), bulimia nerviosa $(\mathrm{BN})$ y trastorno por atracón (TA). Las alteraciones alimentarias significativas que no cumplen los criterios para estos diagnósticos se han incluido dentro del grupo Trastornos especificados y no especificados de la ingesta y la conducta alimentaria

Teniendo en cuenta el duro impacto que puede ocasionar esta patología se han ido desarrollando numerosos estudios epidemiológicos que intentan medir su alcance (Gustafsson, Edlung, Kjellin y Norring, 2009). Los estudios epidemiológicos sobre AN en España, empiezan a surgir a partir de 1990. Los más recientes, ponen de manifiesto que la prevalencia aproximada de la AN es $0,9 \%$ en mujeres y 0,3\% en hombres (Álvarez-Malé, Bautista y Serra, 2015; Martinez y De Irala, 2003; Olesti, et al., 2008; Peláez, Labrador y Raich, 2004).

En la etiopatogenia de esta enfermedad intervienen tanto factores genéticos, como biológicos, psicológicos, sociales y culturales. Todos ellos actúan como factores predisponentes (riesgo), desencadenantes (precipitantes) o mantenedores del TCA, considerándose en la actualidad un trastorno multifactorial (Madruga, Leis y Lambruschini, 2012).

Algunos de los factores que pueden precipitar el inicio del trastorno son; trastornos afectivos, trastornos de la personalidad, críticas peyorativas del entorno hacia el cuerpo de la persona, el inicio de las dietas, las fluctuaciones en el peso, los cambios corporales (sobre todo en la etapa de la adolescencia), acontecimientos vitales (ruptura amorosa, separación de los padres...) y enfermedades médicas, sobre todo las que implican una pérdida de peso (Santana, Junior, Giral y Raich, 2012).

Respecto a los factores de riesgo para TCA, éstos se clasifican en tres categorías: individual, psicosocial y sociocultural (Schiltz, 1997). Como factores individuales encontramos: experiencias de abuso sexual, insatisfacción con la figura física, insatisfacción con el peso corporal, práctica crónica de dietas, pérdida de un ser querido, ruptura de una relación importante, divorcio de los padres, cambio geográfico en la zona de residencia y estudio; como factores psicosociales: trastornos afectivos en el individuo y/o en familiares de primer grado, necesidad de aprobación, dificultad para expresar emociones, presencia constante de ira, evasión de conflictos, patrón de pensamiento rígido, sensación de ausencia de autocontrol, perfeccionismo, problemas básicos de identidad; y como factores socioculturales: ideales culturales de belleza, actitudes de la familia hacia el peso corporal, falta de definición de límites y rigidez y confusión de roles en la familia. 
Como factores mantenedores de la enfermedad se encuentran las alteraciones psicológicas, el aislamiento social, la falta de apoyo familiar, la malnutrición, las crisis familiares y la interacción social, en la cual el entorno del paciente refuerza la pérdida ponderal (Madruga et al., 2010).

Los TCA muchas veces se acompañan de complicaciones médicas, así como psicológicas, que potencialmente pueden dejar secuelas irreversibles para el desarrollo del adolescente. Para evitar la cronicidad del trastorno, se recomienda un tratamiento temprano lo cual requiere de un diagnóstico y derivación oportunos a un equipo especializado (López y Treasure, 2011).

El tratamiento de los Trastornos Alimentarios puede llevarse a cabo desde distintos niveles de recursos asistenciales en función de la gravedad de los mismos, intervenciones a nivel ambulatorio, centro de día o ingreso hospitalario (Pike et al., 2003).

Desde un abordaje psicológico, distintas aproximaciones terapéuticas han mostrado su eficacia en el tratamiento de los TCA. En el caso de la Anorexia Nerviosa, la APA (American Psychiatric Association, 2000) establece que la Terapia Cognitiva-Conductual (TCC) es el tratamiento más investigado y con mayor apoyo empírico para su tratamiento.

Concretamente, la terapia cognitivo-conductual, aplicada a los TCA, permite trabajar en profundidad los pensamientos distorsionados sobre la figura, el peso y la alimentación, y lo logra a través de la normalización de los patrones alimentarios, la reestructuración cognitiva y la exposición de los pacientes a situaciones de riesgo y miedos irracionales (Lock, 2005).

\section{Descripción del caso}

\section{Identificación paciente}

Adolescente de 13 años, diagnosticada anorexia nerviosa restrictiva, que acude junto a sus padres quienes son los que solicitan tratamiento.

La paciente es hija única y vive en el domicilio familiar junto a sus padres. Cursa $2^{\circ} \mathrm{ESO}$ y su rendimiento académico es altamente satisfactorio.

\section{Motivo de consulta}

Los padres acuden junto a su hija alarmados frente al deterioro físico tan elevado de los últimos de 6 meses. Refieren estar desconcertados al no saber qué es lo que le está ocurriendo a su hija. Comentan que aproximadamente un año antes, la paciente comienza a bajar de peso, no dándole importancia a este hecho. Sin embargo, cuando el descenso del peso seguía produciéndose es cuando comenzaron a observar que su hija descartaba alimentos de sus ingestas, a lo que la paciente respondía que el motivo era el deseo de "cuidarse".

El peso de la paciente en la primera sesión de evaluación psicológica era de $38,2 \mathrm{~kg}$. El valor del IMC resultante entre su peso y altura era de 14.74. Se descartó patología o enfermedad física mediante las exploraciones médicas pertinentes.

\section{Evaluación del caso}

Se extendió durante las primeras 2 sesiones, con una periodicidad semanal. Para ello se hizo uso de diversas herramientas: 
Entrevista semiestructurada: las áreas evaluadas son: motivo de consulta, peso corporal, conducta alimentaria, imagen corporal, tratamientos previos, historial médico, historia familiar, historia social y del desarrollo, y síntomas comórbidos.

Así mismo, se emplearon una serie de cuestionarios, que podrían dividirse en dos grupos. Aquellos específicos para la evaluación de los TCA:

Test de Actitudes Alimentarias (Eating Attitudes Test, EAT) (Gardner y Garfinkel, 1979). El EAT fue diseñado para la evaluación de las actitudes alimentarias anómalas, en especial las relacionadas con el miedo a ganar peso, el impulso de adelgazar y la presencia de patrones alimentarios restrictivos. El EAT es una herramienta autoaplicada de 40 ítems (EAT-40). Cada ítem se valora en una escala Likert de 6 puntos, que van desde nunca a siempre. En la adaptación española realizada por Castro, Toro, Salamero y Guimerà (1991), la consistencia interna entre sus ítems es de $\alpha=.93$.

Cuestionario de Bulimia Revisado (Bulimia Test-Revise BULIT-R) (Smith, Thelen, Farmer y Wonderlich, 1991). El BULIT fue diseñado con el objetivo de cubrir algunas necesidades detectadas en el ámbito de la evaluación de la BN, como son la distinción entre personas con $\mathrm{BN}$ frente a personas sin problemas de TCA; personas con $\mathrm{BN}$ frente a personas con otros TCA, y subgrupos de BN basados en criterios específicos. La versión revisada, su aportación más importante, es la adaptación a los criterios del DSM-III-R y posteriormente al DSM-IV. Consta de 36 ítems, aunque sólo 28 son utilizados para determinar la puntuación final que oscila entre 28-140 puntos. En la versión española elaborada por Mora y Raich (1993), la fiabilidad del cuestionario es de 0.99.

Escala de Yale Brown-Cornell para Trastornos de Alimentación (YBC-EDS) (Mazure y Sunday, 1995). La escala YBC-EDS para trastornos de la alimentación se ha elaborado con el fin de emplearse como instrumento para evaluar la severidad de la sintomatología, considerando los síntomas de las preocupaciones y los rituales. Explora y define las preocupaciones y obsesiones entorno a los alimentos, con respecto al acto de comer, por la forma o la apariencia corporal, y las conductas compulsivas. En la versión española la consistencia interna del cuestionario es de 0.87 (Sal et al., 2002).

Cuestionario de la forma corporal (Body Shape Questionnaire, BSQ) (Cooper, Taylor, Cooper y Fairburn, 1987). Este cuestionario mide la insatisfacción corporal, el miedo a engordar, la autoestima, y el deseo de perder peso. Administrado con el objetivo de medir la preocupación por el peso y la imagen corporal, la insatisfacción producida por el propio cuerpo, el miedo a engordar, la autodesvaloracion por la apariencia física, el deseo de perder peso y la evitación de las situaciones en las que la apariencia física pueda atraer la atención. La adaptación española presentó una consistencia interna de 0.96 (Raich et al., 1996).

Aquellos instrumentos que abarcan otras áreas de interés para la evaluación psicopatológica se describen a continuación:

Inventario de Depresión de Beck-II (Beck Depression Inventory- II) (Beck et al., 1996). Instrumento de autoinforme que evalúa la presencia y severidad de la depresión en adolescentes y adultos. Valora fundamentalmente los síntomas clínicos de melancolía y los pen- 
samientos intrusivos. Es la escala que mayor porcentaje de síntomas cognitivos presenta, destacando además la ausencia de síntomas motores y de ansiedad. La adaptación española del BDI-II obtuvo un coeficiente alfa de fiabilidad de 0.89 (Sanz, García-Vera, Espinosa y Vázquez, 2005).

Inventario de Ansiedad Estado-Rasgo (State Trait Anxiety Inventory, STAI-A/E) (Spielberger et al., 1970). Instrumento empleado para evaluar la ansiedad en dos dimensiones; la primera, ansiedad estado, hace alusión al estado de ansiedad del momento actual del sujeto, que puede llegar a percibirlo por ejemplo mediante la tensión y la activación del sistema nervioso central. Por otro lado, la ansiedad rasgo se refiere a la tendencia y predisposición de un individuo a interpretar de forma amenazante diversas situaciones, creando así niveles de ansiedad constantes y habituales en la persona. Se ha hecho uso de la adaptación española de Buela-Casal, Guillén-Riquelme y Seisdedos Cubero (2011), la cual presenta una consistencia interna de entre 0,89-0,95 en ansiedad estado (A/E), y entre 0,82-0,91 en ansiedad rasgo (A/R).

\section{Formulación Clínica}

\section{Historia del problema/ Hipótesis de Adquisición}

El inicio de la enfermedad se sitúa un año antes, en el verano del 2016, cuando tal y como refiere la paciente, comienza a sentir insatisfacción y preocupación por su apariencia física. El cuadro clínico comienza con conductas alimentarias restrictivas y muy selectivas, en cuanto a cantidades y tipo de alimento. En cuanto a la actividad física, ésta no es ni intensa ni elevada, practicándola dos veces por semana como un método para control calórico.

En la primera toma de contacto con la paciente, ésta mostró un estado de ánimo plano, con escasas muestras de contenido afectivo. Verbalizó sentirse triste, pero en su comunicación no le acompañaba la intensidad en su expresión. No presentaba tendencia al llanto y en ocasiones, la paciente llegaba a mostrar apariencia de ausencia. Reconocía que a veces se encontraba inmersa en estallidos de enfado y cólera. Mostraba una pérdida de interés hacia el entorno, con una tendencia al aislamiento al no percibir el disfrute con actividades sociales, lúdicas y académicas. La apariencia en todo momento de la paciente era de una intensa falta de energía, con un habla escaso y monosilábico.

A nivel físico la paciente, presentaba una imagen deteriorada con un infrapeso severo, tez apagada, lanugo en extremidades superiores, sequedad en la piel, caída intensa del cabello. Refirió dificultades para mantener un sueño reparador, produciéndose despertares frecuentes. Aumentando la sensación de debilidad y cansancio físico. A nivel cognitivo, se identificó una estructura obsesiva, intensificada por elevados y recurrentes pensamientos obsesivos, con un marcado carácter egosintónico. Escasa conciencia y función reflexiva acerca de su estado. Se descartaron planes de ideación autolítica y/ suicida.

La paciente no presentaba conductas de uso y/o de sustancias tóxicas, ni antecedentes médicos de relevancia.

No menarquia. La paciente, antes de producirse el desarrollo de su anorexia nerviosa, no había alcanzado la suficiente madurez biológica que pudiera provocar tal hito físico. 
A nivel familiar, se percibía un alta cohesión y fusionamiento en su estructura y en su dinámica. El estilo educacional mantenía una tendencia hacia la permisividad y la sobreprotección. Las habilidades de afrontamiento eran escasas; y sus jerarquías estaban poco definidas y el liderazgo era difuso. Las normas, pautas y consecuencias eran imprevisibles y rígidas.

En el ámbito académico, la paciente mostraba actitudes de una alta exigencia e hiperresponsabilidad. Al principio ésta era un área que le producía una gran satisfacción y sentimientos de elevada autoeficacia, reforzado con bastante frecuencia pro su entorno familiar. A medida que la exigencia aumentaba, como producto de su estructura obsesiva y el infrapeso, los estudios comenzaron a provocarle emociones intensas que percibía como desagradables. Dedicaba largas jornadas de estudio, con la sensación de que nunca era suficiente y la probabilidad y el temor a suspender, le parecían como más reales, iniciando un círculo vicioso donde iba añadiendo cada vez más horas de estudio a la vez que el malestar también aumentaba.

El padre mostraba niveles elevados de ansiedad frente al crecimiento y la autonomía propia de la pubertad y la adolescencia de su hija. Mostraba excesivo control, alta cercanía en la emocionalidad de su hija, donde en ocasiones se producía el contagio emocional. La paciente frente a estas actitudes de su padre, desarrollaba emociones de culpa y angustia, adoptando cada vez más actitudes infantiles y conductas de aproximación para rebajar y aliviar la ansiedad de su padre.

\section{Hipótesis de Mantenimiento}

La paciente mantiene una dieta restrictiva y el desarrollo de la Anorexia Nerviosa, promovida por factores externos e internos. Como factores externos, la ansiedad del padre como respuesta a su incipiente desarrollo puberal; y como factores internos la ausencia de un sentido de autonomía y diferenciación frente a la figura de un tercero, en este caso su padre.

El bajo peso le permitió detener su crecimiento y desarrollo físico y psicológico, además de frenar la construcción de su identidad como independiente del medio. De esta manera la angustia del padre no aumentaba, pero dejaba a la paciente en una posición de dependencia física y emocional.

\section{Diagnóstico}

Tras la aplicación y análisis de la información sustraída de la entrevista, los cuestionarios y los autorregistros ad hoc, la paciente recibe el diagnostico (F50) de Anorexia Nerviosa restrictiva [307.1], según los criterios DSM-5 (APA, 2013).

Diseño del estudio

Se trata de un estudio de caso único $(\mathrm{N}=1)$, en el cual se utiliza un diseño sin retirada A-B. Por lo tanto, el individuo es el propio control, y se mide el cambio/efecto que produce el tratamiento sobre los objetivos terapéuticos establecidos.

\section{Intervención}

Objetivo general del tratamiento

- Reducir sintomatología de una paciente con Trastorno de la Conducta Alimentaria a través de una intervención psicológica cognitivo-conductual 
- Objetivos específicos

- Establecimiento de un patrón de alimentación saludable

- Lograr una recuperación ponderal del peso de forma progresiva

- Adquisición y práctica de herramientas y estrategias emocionales

- Reestructuración cognitiva de creencias disfuncionales entorno al peso y comida

- Promover la autonomía emocional de la paciente

- Reducción de la sintomatología ansiosa del padre

- Reestructuración de ideas disfuncionales del padre frente al desarrollo de su hija

- Promover actitudes y conductas de crianza saludables hacia la paciente

\section{Aplicación del tratamiento}

A pesar de que el índice de masa corporal de la paciente indicaba la preferencia por un tratamiento más efectivo en una unidad de ingreso especializada, el tiempo de espera en la valoración y admisión de ésta, motivó que se iniciara tratamiento individual en consultas externas basado en un abordaje cognitivo-conductual (CC). El eje de la intervención terapéutica CC se estructura en las fases propuestas por Garner, Vituosek y Pike (1997) para el tratamiento de la AN.

El contenido de las sesiones a lo largo del tratamiento ha mantenido una estructura similar. Las sesiones comienzan con el pesaje de la paciente y en función del peso, se construye los objetivos de la sesión. Se revisan las tareas fijadas en la sesión anterior, se trabajan los objetivos (en función de la fase de tratamiento en la que se encuentre), para posteriormente hacer un resumen y volver a fijar y marcar metas para la próxima sesión. La estructura de las sesiones contiene: Pesaje; valoración y discusión del peso con la paciente en función de las metas fijadas; revisión del autorregistro alimentario y su patrón alimentario; valoración y reestructuración de esquemas y creencias disfuncionales y/o trabajo de temas terapéuticos (en función de la fase); resumen de la sesión y resolución dudas; tareas y ejercicios para casa.

\section{Fase I. Construyendo la confianza y el marco adecuado para el tratamiento}

El objetivo de esta primera fase fue la de trabajar para aumentar la motivación de la paciente para el cambio. Que la paciente adquiriera conciencia sobre su estado fue de vital importancia para poder introducir las primeras intervenciones psicoeducativas.

Esta fase estuvo activa al menos 8 meses, ya que la paciente mostraba una alta resistencia propia de su estructura obsesiva. Los temas tratados en esta etapa, se mantenían abiertos con la posibilidad de volver a retomarlos en cualquier momento en función de la necesidad de la paciente.

Es este sentido, la alianza terapéutica fue la que permitió seguir progresando hacia los objetivos. En los primeros estadios del tratamiento, la discusión y refutación cognitiva no fue 
la herramienta de elección. La validación de la emoción y su expresión, como resultante de los esquemas disfuncionales, es la que nos permitió que la paciente adoptara una actitud de confianza. La postura terapéutica se alejó de estilos más directivos, fomentando la expresión del malestar sin perder la neutralidad.

Se transmitió gran cantidad de información con el objetivo de ir dotando a la paciente cierta sensación de control sobre conductas de salud y sobre su malestar emocional.

Los temas tratados durante esta fase no siguieron un orden establecido, pudiendo volver a ellos si se consideraba necesario para que la paciente pudiera ir logrando las metas y los objetivos. Los contenidos de los temas son los siguientes: construcción alianza y confianza en el terapeuta; psicoeducación sobre los síntomas de desnutrición y el infrapeso; valoración, tratamiento y adquisición de papel activo frente a las consecuencias físicas; valoración, tratamiento y adquisición de papel activo frente a las consecuencias psicosociales; planteamiento de las hipótesis sobre la funcionalidad de la sintomatología; establecimiento de los objetivos del tratamiento; estimulación del razonamiento lógico cognitivo; aumentar tolerancia hacia la recuperación del peso ponderal; asegurar patrón alimentario saludable sin restricción y sin exclusión; prevención de atracones y posible vómitos tras la restauración alimentaria; trabajo cognitivo inicial (errores y distorsiones cognitivas); preservar la motivación (objetivo transversal a todas las fases); incrementar la conciencia crítica hacia los valores culturales acerca de la delgadez; psicoeducación familiar (extinguir respuestas entorno familiar como mantenedoras).

A continuación, se describe las técnicas utilizadas en esta primera fase del tratamiento:

1. Psicoeducación: el objetivo principal de esta técnica fue la de dar una visión realista de las consecuencias que la $A N$ tiene en la vida de las personas, por un lado, describir las consecuencias a nivel físico y emocionales e interpersonales de la enfermedad. Se transmitió la idea de que tener un TCA es incompatible con la vida que uno espera tener. Para el apoyo de este objetivo, se utilizó un vídeo de una paciente adulta de larga evolución donde el deterioro físico, emocional e interpersonal estaba muy comprometido.

Síntomas de inanición. Para ello se aplicó el Estudio de Minnesota (Keys, Brozek, Henschel, Mickelsen y Taylor., 1950) cuyo es el de atribuir mucha de la sintomatología de la AN al estado actual de inanición.

Peso. Se introdujo el concepto de IMC como indicador de salud. La explicación del peso, se alejó del criterio numérico, centrándose en cuestiones sobre peso natural, la funcionalidad del peso natural y el mantenimiento del peso natural, peso ideal, los componentes biológicos de la cifra numérica (masa muscular, masa proteica, masa ósea, masa grasa...).

Métodos inadecuados. Se explicó la psicoeducación sobre las consecuencias físicas y emocionales del inicio y mantenimiento de las dietas, y la necesidad de la eliminación de la misma y de otro tipo de dietas basadas en la ingesta de poca cantidad de comida o de un determinado número fijo e inflexible de calorías diarias., la exclusión de ciertos alimentos considerados como calóricos o muy calóricos. 
Nutrición. En este punto se le explicó la importancia de los nutrientes y los cuatro grupos de alimentos, los cambios en los requerimientos de energía, la distribución de la proporción de nutrientes, el balance energético y el gasto metabólico necesario, y la elaboración de menús.

Explicación de las múltiples funciones de la sintomatología de la anorexia. Se presentó a la paciente el marco conceptual sobre las múltiples funciones adaptativas que su AN mostró. La explicación se desarrolló a través de un análisis funcional de los refuerzos positivos y negativos como mantenedores de su TCA.

2. Tareas de motivación al cambio

Dirigidas a las conductas de restricción calórica y alimentaria, vivenciadas por la paciente como egosintónicas. Las tareas incluidas son las siguientes:

Ejercicio "Querida Anorexia”. El objetivo de este ejercicio fue que la paciente empezara a distanciarse cognitivamente de la Anorexia, la represente como algo externo a ella. Se trata que la paciente entienda que su TCA ha tenido una serie de funciones en su vida y que es normal que se aferre a este trastorno. De esta manera logramos desactivar todo el oposicionismo y la resistencia que genera el creer que le vamos a "quitar" el TCA.

Análisis de aspectos positivos y negativos de tener un trastorno alimentario. El objetivo fue el identificar la otra visión de la paciente del sufrimiento, las interferencias y las consecuencias negativas que su trastorno ha generado en su vida.

Proyectándonos en el futuro. Se persiguió la reflexión de la paciente sobre la funcionalidad de sus comportamientos alimentarios actuales para controlar el peso si estos persistían en el tiempo.

3. Técnicas conductuales

Autoobservación y autorregistro alimentario. El objetivo es que la paciente adquiera información acerca de su problema alimentario, y que este aumento en la conciencia genere ayuda al cambio.

Instauración del patrón alimentario y normalización de la ingesta. El objetivo es que la paciente realice las 5 ingestas, que incremente la cantidad de comida ingerida, que amplíe el rango de comida ingerida y la eliminación de conductas compensatorias.

4. Técnicas de exposición

- Explicación de la introducción de alimentos prohibidos (AP). El objetivo es introducir aquellos alimentos "prohibidos" para la paciente en su vida diaria. A continuación, se describen los pasos a seguir:

- Evaluar las consecuencias positivas y negativas de la evitación de los alimentos "prohibidos"

- Analizar cómo enfrentarnos a este problema: exposición, introducción de los AP

- Explicación de la técnica de exposición

- Construir, revisar y corregir una jerarquía de exposición de los AP

- Planificación de la introducción de 2 AP semanales 
Explicación de la técnica de exposición a las zonas corporales temidas por la paciente. Se le explica las consecuencias negativas de la evitación, el objetivo de la propia exposición y sus principios. Tras la explicación, la paciente junto con el terapeuta confecciona las jerarquías de exposición (jerarquías de exposición a zonas corporales, al cuerpo desnudo y a las situaciones de imagen corporal).

Fase II. Cambio de las creencias asociadas al cuerpo y a la comida. Ampliando los objetivos de la terapia

El objetivo que se persiguió en esta fase fue la de proseguir con el aumento de peso, ampliando además los contenidos de la terapia hacia el cambio de actitudes y creencias entorno al cuerpo y peso. Se promovió además el fortalecimiento y mejora del autoconcepto de la paciente.

Se cuestionaron por tanto el autoconcepto y los esquemas de perfeccionismo que la paciente aplicaba a las definiciones y juicios que hacía de sí misma, dotándole de herramientas más adaptativas en la manera de percibirse y valorarse.

En esta fase, promueve la adquisición y la practicas de habilidades de resolución de conflictos personales e interpersonales. Se le enseño, además, a tomar conciencia emocional y a manejar la tolerancia a emociones desagradables; para que lograra una gestión emocional eficaz.

A nivel cognitivo, se trabajó las creencias disfuncionales acerca de la estética dominante y que eran un elemento mantenedor de su trastorno.

En lo que respecta a la conducta alimentaria, se fomentó la flexibilidad y la espontaneidad en la selección del alimento; y la normalización de las cantidades en las tomas alimentarias. Las técnicas empleadas en esta fase se explican a continuación.

1. Técnicas de reestructuración cognitiva

Introducción a la técnica de reestructuración cognitiva (TREC). Se utilizan las técnicas clásicas de reestructuración cognitiva (Beck, 1976).

Práctica de las habilidades aprendidas con la técnica TREC sobre las creencias de la AN

Revisión del autoconcepto mediante la TREC. Utilizando las técnicas cognitivas aprendidas se revisa las metas vitales de la paciente. El objetivo es hacerle consciente que mantener unas metas rígidas, inflexibles y perfeccionistas le llevan al continuo fracaso, en el área social, académica y emocional. También se le hace consciente que esta meta única, rígida e inflexible es en la que basa todo su autoconcepto y autoestima

2. Técnicas de solución de problemas (SP) (D’Zurilla y Golfried, 1971)

Introducción a la técnica de SP. Se plantea esta técnica como modo de afrontamiento de situaciones problemáticas, ya que la paciente bajo situaciones de estrés emocional recurre al síntoma de la restricción.

Práctica de la técnica de SP en la vida de la paciente. Se le pide que ponga en práctica 
en su vida diaria, siguiendo las fases de la técnica de SP: orientación general hacia el problema; definición y formulación del problema; generación de soluciones alternativas; toma de decisiones; puesta en práctica y verificación de la solución.

3. Técnicas de exposición

\section{Introducción Alimentos "Prohibidos"}

Exposición a las zonas corporales evitadas por la paciente

4. Intervención en otros temas. Se interviene en temas que son relevantes para la paciente y que no tienen que ver con el peso (reconocimiento e identificación de emociones, manejo impulsividad emocional, habilidades sociales, toma de decisiones).

Fase III. Prevención de recaídas y preparación del fin de la terapia.

El objetivo de esta fase es la de consolidar los logros obtenidos en los meses anteriores. Se fomenta además la tendencia de atribución interna de los logros y la resolución de conflictos. Se le enseña a distinguir una "caída" de una recaída y se repasan los logros consolidados y las estrategias que le han resultado más útiles. Se repasan los factores de riesgo y se prepara un plan de acción en caso de que surjan.

A nivel familiar, la intervención con los padres se sustenta en técnicas de psicoeducación y en herramientas de expresión emocional, con el objetivo de adquirir las habilidades de afrontamiento al TCA de su hija. Concretamente con el padre, la reestructuración cognitiva, se emplea como técnica con el fin de lograr un cambio en su sistema de creencias entorno al crecimiento de su hija, y adoptar una postura funcional y un rol en la dinámica familiar acorde con la etapa de desarrollo de su hija adolescente, que le permitiera relacionarse con la distancia emocional necesaria sin obstaculizar el proceso de diferenciación de su hija.

En cuanto al tratamiento médico, su psiquiatra de referencia le prescribe Tranxilium Pediátrico: $0-0-2,5 \mathrm{mg}$.

\section{Resultados}

Una vez efectuada la evaluación pre y post tratamiento, se exponen los siguientes resultados Análisis cuantitativo:

- EAT

De este cuestionario la puntuación obtenida por la paciente en la evaluación inicial asciende a 76. En la fase POST, la paciente obtiene una puntuación directa de 28.

\section{- BULIT-R}

En la fase PRE la puntuación obtenida por la paciente asciende a 70; y en la post evaluación, los resultados obtenidos son de 52.

\section{- YBC-EDS}

En la evaluación inicial las ideas, los pensamientos e imágenes en torno a los alimentos, al acto de comer, al peso y a la actividad física son recurrentes y la paciente las vive como egosintónicas, otorgándoles una categoría de realidad y veracidad. Su conciencia y su postura crítica muestran niveles mínimos. 
Estas cogniciones ocupan y aparecen con elevada frecuencia, generándole gran preocupación y la necesidad de activar rituales y estrategias para paliar el malestar asociado.

Concretamente, mantiene una clasificación muy exhaustiva de lo que considera alimentos buenos y malos; además de presentar un componente fóbico en el acto de comer en público.

En cuanto a las conductas de compulsión, la paciente siente la necesidad de poner en marcha rituales como la necesidad de cortar cada pedazo del alimento en un tamaño específico, la de consumir cada ingesta en un orden concreto o la de dejar de forma invariable en el plato algo de comida. Muestra, conductas de higiene y limpieza después de cada bocado de comida.

- BDI-II

De los resultados iniciales se desprende una elevada sintomatología depresiva, puntuación de 32 (Depresión grave). Mientras que en la evaluación post, los resultados descienden 18 puntos, obteniendo una puntuación directa de 14 (Depresión leve).

- STAI-A/E

En la evaluación de ansiedad estado inicial, la puntuación directa es 80. En la fase POST, la puntuación sufre un descenso de 15, obteniendo un total de 65 .

En la fase PRE, la puntuación directa de la ansiedad rasgo al inicio fue de 70. La evaluación final desciendo 10 puntos, alcanzando una puntuación directa de 60 .

- BSQ

En esta medida al inicio de la evaluación el resultado ascendía a una puntuación directa de 133.

En la fase POST, se observa un descenso significativo en el resultado, mostrando una puntuación de 76.

\section{Medidas Físicas}

En la fase PRE, el peso inicial de la paciente es de 38,2kg y un IMC de 14.74 (Delgadez severa). Tras el tratamiento, la intervención psicológica y la post evaluación, la paciente presenta un peso de 49,2kg y un IMC de 18.9 (Peso normal).

\section{Análisis cualitativo}

Analizar la motivación del paciente a través de la adherencia a la asistencia a las sesiones y las cancelaciones puede indicar el aumento y el mantenimiento de un compromiso frente al tratamiento. La paciente ha mantenido durante todo el tratamiento actitudes positivas hacia las sesiones y hacia los objetivos de las mismas. Además, el contenido de las verbalizaciones se modificó desde la negación al cambio a deseos verbalizados de cambio. La cumplimentación de las tareas tras las sesiones, mostró una alta voluntariedad hacia el trabajo terapéutico. Se observó una modificación en las creencias entorno al peso, la comida y el cuerpo a través de una comunicación más flexible y menos crítica.

En lo relativo al patrón alimentario, la paciente logra introducir una conducta alimentaria más saludable. La paciente incorpora a la alimentación aquellos alimentos que consideraba 
prohibidos en una frecuencia normalizada, gracias al cambio de las creencias disfuncionales y mitos alimentarios.

La paciente tras el tratamiento, incorpora en su autoevaluación aspectos que van más allá de la delgadez, logrando aumentar la sensación de eficacia percibida. Para ello, tomó un papel activo, aumentando el número de actividades agradables y siendo capaz de atribuir valía a estas otras áreas de desarrollo personal.

El estado de ánimo depresivo y ansioso disminuye. Este cambio se percibe en los comportamientos no verbales, los cuales adquieren energía y sintonía con el ambiente y con el otro; las verbalizaciones son optimistas y esperanzadoras, visualizándose en un futuro a corto, medio y largo plazo sin la enfermedad y poniendo en marcha estrategias de afrontamiento activas en la consecución de objetivos deseados.

A nivel físico, la renutrición y el restablecimiento del peso, permitió que el desarrollo puberal continuara y se produjera la menarquia.

A nivel familiar, se inició el proceso de diferenciación y autonomía tanto emocional como física, logrando la paciente establecer lazos vinculares de independencia en lo que tiene que ver con su mundo interno cognitivo y emocional. El padre logró reducir los niveles de ansiedad, además de percibir el desarrollo de su hija como un hito necesario y empezó a mostrar actitudes y herramientas psicológicamente más saludables frente al desarrollo de su hija.

\section{Discusión}

Para el abordaje de este caso se ha llevado a cabo una intervención cognitivo- conductual, propuesta por Garner et al. (1997), para el tratamiento de una Anorexia Nerviosa según criterios DSM-5 (APA, 2013).

Se establecieron una serie de objetivos tras la evaluación y la formulación del caso (análisis funcional (AF)). Tras la elección de las técnicas de intervención en función del AF, la planificación del tratamiento y la aplicación del mismo, se valoran los siguientes resultados del tratamiento.

La efectividad hace referencia al grado en que un tratamiento logra los objetivos terapéuticos, y la orientación cognitivo comportamental permitió el restablecimiento de un patrón alimentario saludable alejado de los síntomas restrictivos que presentaba la paciente lo que facilitó la progresión de la recuperación ponderal del peso. Además, se observó la mejoría de la paciente en aquellas áreas que se establecieron como objetivos del tratamiento. A nivel cognitivo, la paciente logró una modificación y reestructuración de creencias e ideas irracionales entorno al cuerpo y la comida, lo que se tradujo en conductas de autocuidado y de salud. A nivel emocional, se alcanzó una mayor conciencia y reflexividad emocional, que le permitió la identificación emocional, la tolerancia emocional y estrategias de gestión emocional. En el ámbito social, la paciente retomó las relaciones con sus iguales, incluso ampliando su círculo social.

La eficacia hace referencia a los logros de los objetivos terapéuticos en condiciones propias 
de la investigación. Al tratarse de un diseño de caso único y no poder valorar comparaciones, esta propiedad psicométrica no pudo evaluarse. Sin embargo, la tendencia de la literatura es la de confirmar la intervención cognitivo-comportamental como el tratamiento de primera elección para la anorexia nerviosa (APA, 2000).

La puesta en práctica de las técnicas aprendidas por la paciente, fue amplia y generalizándose a la mayoría de los contextos y ambientes de su día a día.

Este trabajo cuenta con una serie de limitaciones que han de ser mencionadas. Una de las principales limitaciones fue el infrapeso de la paciente que imposibilitó la intervención cognitiva en la etapa inicial.

El estado físico inicial requirió el restablecimiento de una alimentación completa que permitiera una recuperación ponderal del peso que permitiera a la paciente encontrarse en un estado físico, emocional y cognitivo mínimo para iniciar el trabajo terapéutico. Por este motivo, las técnicas y medidas conductuales se intensificaron y se alargaron temporalmente, retrasándose la intervención cognitiva.

La paciente cumplía los criterios necesarios para un ingreso hospitalario, sin embargo, el procedimiento se extendió temporalmente, y se inició la intervención terapéutica a la espera de la derivación hospitalaria. En este intervalo de tiempo, la paciente empezó a responder a las pautas y medidas conductuales, realizando una recuperación ponderal del peso y los objetivos propuestos para su caso, logrando un estado físico y emocional que no revestían la gravedad para el ingreso en una unidad de Trastornos de Conducta Alimentaria.

\section{Referencias}

American Psychiatric Association (APA). (2000). Practice guideline for the treatment of eating disorders. Washington, DC: APA.

American Psychiatric Association (APA). (2013). Diagnostic and Statistical Manual of Mental Disorders-5. Washington, DC: APA.

Álvarez-Malé, M., Bautista, I. y Serra, LL. (2015). Prevalence of eating disorders in adolescents from Gran Canaria. Nutrición Hospitalaria, 31(5), 2283-2288.

Beck, A. (1976). Cognitive Therapy and the Emotional Disorders. New York: International University Press.

Beck, A., Steer, R. y Brown, G. (1996). Manual for the Beck Depression Inventory-II. San Antonio, TX: Psychological Corporation.

Buela-Casal, G., Guillén-Riquelme, A. y Seisdedos-Cubero, N. (2011). Cuestionario de ansiedad estado-rasgo. Octava edición. Madrid: TEA.

Castro, J., Toro, J., Salamero, M., \& Guimerá, E. (1991). The Eating Attitudes Test: Validation of the Spanish version. Evaluación Psicológica, 7(2), 175-189.

Cooper, P., Taylor, M., Cooper, Z., y Fairburn, C. (1987). The development and validation of the Body Shape Questionnaire. International Journal of Eating Disorders, 6(4), 485-495.

D'Zurilla, T., y Goldfried, M. (1971). Problem solving and behavior modification. Journal of Abnormal Psychology, 78, 107126.

Garner, D. y Garfinkel, P. (1979). The Eating Attitudes Test: An index of the symptoms of anorexia nervosa. Psychological 
Medicine, 9, 273-279.

Garner, D., Vitousek, K. y Pike, K. (1997). Cognitive-Behavioral Therapy for AN. En Garner y Garfinkel (Eds.), Handbook of treatment for eating disorders. New York: Guilford Press.

Graell, M., Villaseñor, A., y Morandé, G. (2014). Clínica y evaluación de los trastornos de alimentación de la edad prepuberal. En Morandé, G., Graell, M., Blanco, M. A. (Coords.) Trastornos de la conducta alimentaria y obesidad. Un enfoque integral. Madrid: Panamericana.

Gustaffson, S., Edlund, B., Kjellin, L. y Norring, C. (2009). Risk and protective factors for disturbed eating in adolescent girls-aspects of perfectionism and attitudes to eating and weight. European Eating Disorders Review, 17 (5), 380 389.

Keys, A., Brozek, J., Henschel, A., Mickelsen, 0. y Taylor, F. (1950). The biology of human starvation. Minnneapolis: University of Minnesota Press.

Lock, J. (2005). A Comparison of Short- and Long-Term Family Therapy for Adolescent Anorexia Nervosa. Journal of the American Academy of Child \& Adolescent Psychiatry, 44, 632-639.

López, C. y Treasure, J. (2011). Trastornos de la conducta alimentaria en adolescentes: Descripción y manejo. Revista Médica Clínica Las Condes, 22(1), 85-97.

Madruga, A., Leis, T. y Lambruschini, F. (2010). Trastornos del comportamiento alimentario: Anorexia nerviosa y bulimia nerviosa. En: Asociación Española de Pediatría \& Sociedad Española de Gastroenterología, Hepatología y Nutrición Pediátrica. Protocolos de Gastroenterología, Hepatología y Nutrición. España: Ergón.

Martínez, M. A. y De Irala, J. (2003). Los trastornos del comportamiento alimentario en España: ¿estamos preparados para hacerles frente desde la salud pública? Gaceta Sanitaria, 17(5), 347-350.

Mazure, C. y Sunday S. (1995). The Yale-Brown-Cornell Eating Disorder Scale: Development, Use, Realiability and Validity. Journal of Psychiatry Research, 28,425-445.

Mora, M. y Raich, R. (1993). Adaptació del bulimia test i BSO en una mostra universitaria. Comunicación presentada en la VIII Jornada de Terapia del Comportamiento y Medicina Conductual en la Práctica Clínica. Barcelona.

Morandé, G., Graell, M., y Blanco, M. (2014). Trastornos de la conducta alimentaria y obesidad. Editorial Panamericana. New York: Guilford Press.

Olesti M., Martín, N., De la Fuente, M., Riera, A., Bofarull J. y Ricoma, G. (2008). Prevalencia de la Anorexia Nerviosa, bulimia nerviosa y otros TCA en adolescentes femeninas de Reus. Anales de Pediatría, 68(1), 18-23.

Ortiz-Moncada, R. (2015). Epidemiología de los trastornos del comportamiento alimentario. Alicante: Universidad de Alicante. Departamento de Enfermería Comunitaria, Medicina Preventiva y Salud Pública e Historia de la ciencia.

Peláez-Fernández, M., Labrador, F. y Raich, R. (2004). Prevalencia de los trastornos de la conducta alimentaria: consideraciones metodológicas. International Journal of Psychology and Psychological Therapy, 5, 135-148.

Peláez-Fernández, M., Ruiz-Lázaro, P., Labrador, F. y Raich, R. (2014). Validación de Eating Attitudes Test como instrumento de cribado de trastornos de la conducta alimentaria en población general. Medicina Clínica, 142(4), 153-155.

Pérez-Gaspar, P., Grual, P., De Irala, J., Martínez-González, M., Lahortiga, F. y Cervera, S. (2000). Prevalencia de los trastornos de la conducta alimentaria en las adolescentes navarras. Medicina Clínica (Barcelona), 144, 481-486.

Pike, K., Wilfiey, D., Hubert, A., y Fairburn, C. (2006). Antecedent life events of binge-eating disorder. Psychiatry Research, 142, 19-29.

Pike, K., Walsh, B., Vitousek, K., Wilson, G., y Bauer, J. (2003). Cognitive behavior therapy in the posthospitalization treatment of anorexia nervosa. American Journalist Psychiatry, 160 (11), 2046-9.

Raich, R., Mora, M., Soler, A., Avila, C., Clos, I. y Zapater, L. (1996). Adaptación de un instrumento de evaluación de la insatisfacción corporal. Clínica y Salud, 7, 51-66.

Ruiz-Lázaro, P., Alonso, J., Comet, P., Lobo, A. y Velilla, M. (2005). Epidemiology of Eating Disorders in Spain. In: Swain PI, Editor. Trends in Eating Disorders Research. New York: Nova Publishers, 85-108.

Sal y Rosas H., Vega-Dienstmaier J, Mazzotti G, Vidal H. y Guimas B, (2002). Validación de una versión en español de la Escala Yale - Brown para el trastorno obsesivo-compulsivo. Actas Españolas Psiquiatría, 30, 30-5. 
Santana, M., da Costa R, H., Mora, M., y Raich, R. (2012). La epidemiología y los factores de riesgo de los trastornos alimentarios en la adolescencia: una revisión. Nutrición Hospitalaria, 27 (2), 391-401.

Sanz, J., García-Vera, M., Espinosa, R., Fortín, M., y Vázquez, C. (2005). Adaptación española del Inventario para la Depresión de Beck-II (BDI-II): 3. Propiedades psicométricas en pacientes con trastornos psicológicos. Clínica y Salud, 16(2), 121-142.

Schiltz, T. (1997). Eating Concerns Support Group Curriculum. Greenfield. Community Recovery Press.

Smith, M., Thelen, M., Farmer, J., y Wonderlich, D. (1991). A revision of the bulimia test: the BULIT-R. Journal of Consulting Clinical Psychological, 3, 119-124.

Spielberger, C., Gorsuch, R. y Lushene, R. (1970). STAI. Cuestionario de Ansiedad Rasgo-Estado. Madrid: TEA. 